\title{
Eighteen polymorphic microsatellite markers for the highly endangered Spanish imperial eagle (Aquila adalberti) and related species
}

\author{
BEGOÑA MARTÍNEZ-CRUZ, * VICTOR A. DAVID, † JOSÉ A. GODOY,* JUAN J. NEGRO,* \\ STEPHEN J. O’BRIEN† and WARREN E. JOHNSON† \\ *Estación Biológica de Doñana (CSIC), Avda. María Luisa s/n, 41013 Sevilla, Spain, †Laboratory of Genomic Diversity, NCI-FCRDC, \\ Frederick, MD, 21702-1201, USA
}

\begin{abstract}
Here we describe the development of 18 polymorphic microsatellite markers for the endangered Spanish imperial eagle (Aquila adalberti). Microsatellites were tested in five other raptor species. These markers were revealed as good molecular tools for genetic population studies, individual identification and parentage assessment in Spanish imperial eagle and closely related species.
\end{abstract}

Keywords: Aquila adalberti, individual identification, microsatellites, paternity assessment, population genetics, Spanish imperial eagle

The Spanish imperial eagle (Aquila adalberti) is one of the most endangered raptors in the world (classified in the CITES Appendix I). During the 20th century, the species has suffered a strong demographic bottleneck (González et al. 1989). Currently, around 131 breeding pairs (year2000 census by SEO Bird Life) are distributed into several breeding nuclei in southwest Spain (Ferrer 1993). The present situation of the species remains critical mainly because of human pressures and the sharp decline of rabbit populations, which are their main prey. The development of molecular genetic tools is deemed crucial for the management and conservation of this species.

Microsatellites are particulary suitable for studying endangered species as they allow working with samples collected noninvasively, such as feathers and eggshells (Pearce et al. 1997; Taberlet et al. 1999; Strausberger \& Ashley 2001). To our knowledge, no microsatellite markers have been described for any Aquila species and they have only been developed for three other raptor species (Nesje \& Roed 2000a; Gautschi et al. 2000; Nesje et al. 2000b). Here we characterize 18 polymorphic microsatellite markers developed for the Spanish imperial eagle. They will allow the assessment of the genetic structure and variability of remaining populations (Nesje et al. 2000c).

Correspondence: Begoña Martínez-Cruz. Fax: +34 9546211 25; E-mail: bemar@ebd.csic.es
Microsatellites were isolated from a GT dinucleotide repeat enriched genomic library using a modified nonradioactive capture-hybridization method (Refseth et al. 1997) modified in Sarno et al. (2000). Genomic DNA was extracted from a blood sample of a female chick Spanish imperial eagle sampled in Aceuche (Cáceres) in 1996, using a standard phenol-cloroform protocol (Sambrook et al. 1989). Microsatellite isolation procedure was as described in Sarno et al. (2000).

Nonredundant clones were selected from fragments that contained only one uninterrupted microsatellite composed of at least 10 tandem repeats and that had flanking sequences that were long enough to design primers. Fortyfour primer pairs were designed from selected clones using the Microsatellite Target Identification Program (R. Stevens and V.A. David, unpublished) and the program primer 3 (available at www.genome.wi.mit.edu/cgibin/ primer/primer3_www.cgi). From an initial amplification in 15 unrelated individuals using dUTP labelling (David \& Menotti-Raymond 1998), 17 polymorphic dinucleotide and one tetranucleotide were selected. Forward fluorescently labelled markers were tested further in a total of 38 Spanish imperial eagles and in other raptor species. Polymerase chain reactions (PCRs) were carried out in a MJ Research PTC-100 thermocycler in $20 \mu \mathrm{L}$ containing $16 \mathrm{~mm}$ (NH4)SO4, $2.5 \mathrm{~mm} \mathrm{MgCl}_{2}, 0.25 \mathrm{~mm}$ of each dNTP, $0.5 \mathrm{U}$ Taq DNA polymerase (Bioline), $0.25 \mu \mathrm{m}$ of each primer and $62.5 \mathrm{ng}$ DNA, under the following conditions: an initial denaturation 
Table 1 Characterization of 18 A adalberti microsatellite loci in 38 individuals from several breeding nuclei

\begin{tabular}{|c|c|c|c|c|c|c|c|c|c|}
\hline \multirow[b]{2}{*}{ Locus } & \multirow{2}{*}{$\begin{array}{l}\text { Genbank } \\
\text { accession } \\
\text { numbers }\end{array}$} & \multirow[b]{2}{*}{ Primer sequences 5'-3' } & \multirow{2}{*}{$\begin{array}{l}\text { Repeat } \\
\text { motif }\end{array}$} & \multirow{2}{*}{$\begin{array}{l}\text { Size } \\
\text { range } \\
\text { (bp) }\end{array}$} & \multirow{2}{*}{$\begin{array}{l}\text { Number } \\
\text { of alleles }\end{array}$} & \multirow[b]{2}{*}{$H_{\mathrm{O}}$} & \multirow[b]{2}{*}{$H_{\mathrm{E}}$} & \multicolumn{2}{|l|}{ HWE } \\
\hline & & & & & & & & $P$-value & $\mathrm{SE}$ \\
\hline Aa02 & AF469500 & $\begin{array}{l}\mathrm{F}^{\wedge}: \text { CTGCAGATTTCACCTGTTCTG } \\
\text { R: CTTCCAGGTCTTGCAGTTTACC }\end{array}$ & $(\mathrm{GT})_{19}$ & $141-155$ & 4 & 0.45 & 0.41 & 0.9046 & 0.0034 \\
\hline $\mathrm{Aa} 04$ & AF469506 & $\begin{array}{l}\mathrm{F}^{\wedge}: \text { TGCAGCTCAAAAGCAAAGG } \\
\mathrm{R}: \text { CAACCCCAACTCTCACACCT }\end{array}$ & $(\mathrm{GT})_{12}$ & $126-130$ & 3 & 0.61 & 0.64 & 0.1025 & 0.0032 \\
\hline Aa11 & AF469497 & $\begin{array}{l}\mathrm{F} \wedge \text { : ACGAGCTTATCTTTGACCAAGC } \\
\text { R: CTTTGTTTTCAGCTGTTCCAGG }\end{array}$ & $(\mathrm{CA})_{11}$ & $246-252$ & 3 & 0.34 & 0.29 & 0.6263 & 0.0051 \\
\hline Aa12 & AF469498 & $\begin{array}{l}\text { F^: TCATCAACCTGACCCTTTCC } \\
\text { R: TGCACTGAAGTTTCTCGGC }\end{array}$ & $(\mathrm{GT})_{12}$ & 133-135 & 2 & 0.40 & 0.50 & 0.2050 & 0.0026 \\
\hline Aa15 & AF469499 & $\begin{array}{l}\mathrm{F}^{\wedge}: \text { TCACTGACCTGCCCTCTACA } \\
\text { R: CCAACCCTCTAGTCGTCCAC }\end{array}$ & $(\mathrm{CA})_{13}$ & 197-203 & 3 & $0.66^{* *}$ & 0.53 & 0.0081 & 0.0009 \\
\hline Аa26 & AF469501 & $\begin{array}{l}F^{\wedge} \text { : GCAAAGGTAAACTGCATCTGG } \\
\text { R: ATGCACTATTGGTAAACAGGCA }\end{array}$ & $(\mathrm{AC})_{14}$ & $145-159$ & 5 & 0.58 & 0.64 & 0.4952 & 0.0124 \\
\hline Аa27 & AF469502 & $\begin{array}{l}\text { F^: GAGATGTCTTCACAGCTTGGC } \\
\text { R: AAGTCTCAGAGACTGACGGACC }\end{array}$ & $(\mathrm{CA})_{11}$ & $92-98$ & 3 & 0.53 & 0.58 & 0.3451 & 0.0052 \\
\hline Aa35 & AF469503 & $\begin{array}{l}\text { F^: GCAGCAGAAAGTGCATACGA } \\
\text { R: GACCAAATGAAATGCGCC }\end{array}$ & $(\mathrm{AC})_{17}$ & $250-264$ & 5 & 0.45 & 0.40 & 0.1408 & 0.0101 \\
\hline Аа36 & AF469504 & $\begin{array}{l}\text { F^: ACAGGCCAGCACCAAGAG } \\
\text { R: TTTGGAGCCATTGTTACCGT }\end{array}$ & $(\mathrm{AC})_{16}$ & 109-119 & 5 & $0.47^{*}$ & 0.62 & 0.0420 & 0.0040 \\
\hline Аa39 & AF469505 & $\begin{array}{l}\text { F^: TTCTGTTTTTCCACTTGCTTG } \\
\text { R: TATTGAGCTCACAAAAACAAAGG }\end{array}$ & $(\mathrm{AC})_{13}$ & $191-223$ & 7 & $0.53 * *$ & 0.77 & 0.0057 & 0.0013 \\
\hline Aa41 & AF469507 & $\begin{array}{l}\text { F^: CCAGCAGGCACCTGTTTTAT } \\
\text { R: AAAAGTTTGGGCATTTGTGG }\end{array}$ & $(\mathrm{CAAA})_{9}$ & 151-159 & 4 & $0.42 *$ & 0.61 & 0.0487 & 0.0041 \\
\hline Aa43 & AF469508 & $\begin{array}{l}\mathrm{F} \wedge \text { : CCACACTGAGAAACTCCTGTTG } \\
\text { R: TTCCTGAGAGCTCTTCCTGC }\end{array}$ & $(\mathrm{AC})_{14}$ & $108-114$ & 4 & $0.18^{* *}$ & 0.30 & 0.0063 & 0.0015 \\
\hline Aa49 & AF469509 & $\begin{array}{l}\mathrm{F}^{\wedge}: \text { AGGAGGTGCCAGTTTTCTCC } \\
\mathrm{R}: \text { AGCGGGTCTGTGGCTCAT }\end{array}$ & $(\mathrm{AC})_{12}$ & $146-156$ & 5 & $0.45 *$ & 0.65 & 0.0183 & 0.0024 \\
\hline Aa50 & AF469510 & $\begin{array}{l}\text { F^: AACATGGCAATGTGTTTCGA } \\
\text { R: ATTGACGCTGCAAACAGATG }\end{array}$ & $(\mathrm{TG})_{11}$ & $209-219$ & 4 & 0.63 & 0.57 & 0.5874 & 0.0074 \\
\hline Aa51 & AF469511 & $\begin{array}{l}\text { F^: CCAGGAAAATGACTGTGGCT } \\
\text { R: GTTCCTGGATGTTCACTTCCA }\end{array}$ & $(\mathrm{TG})_{11}$ & $230-232$ & 2 & 0.42 & 0.47 & 0.7279 & 0.0018 \\
\hline Aa53 & AF469512 & $\begin{array}{l}\text { F^: ATCGCTTCCATGAGCTGATT } \\
\text { R: GAGTGCGGAGAGCTCTGC }\end{array}$ & $(\mathrm{CA})_{12}$ & $123-133$ & 5 & $0.34^{* * *}$ & 0.63 & 0.0001 & 0.0001 \\
\hline Aa56 & AF469513 & $\begin{array}{l}\text { F^: GGGGTGAAACACAGATGCTT } \\
\text { R: CAAGCAACTGGCAACTTGAA }\end{array}$ & $(\mathrm{GT})_{14}$ & $249-263$ & 6 & $0.42 *$ & 0.46 & 0.0493 & 0.0071 \\
\hline Aa57 & AF469514 & $\begin{array}{l}\mathrm{F}^{\wedge}: \text { AACATTAAGGCAGATGTGGACA } \\
\mathrm{R}: \text { TACTGTGGACACGGACAGGA }\end{array}$ & $(\mathrm{TG})_{12}$ & $113-115$ & 2 & 0.29 & 0.32 & 0.6086 & 0.0018 \\
\hline
\end{tabular}

Exact test of Hardy-Weinberg equilibrium showed significant heterozygote deficits in some loci $\left({ }^{*} P<0.05 ;{ }^{* *} P<0.01 ;{ }^{* * *} P<0.001\right)$.

$\wedge$ indicates fluorescently labelled primer.

step at $94{ }^{\circ} \mathrm{C}$ for $2 \mathrm{~min} ; 17$ cycles of $92{ }^{\circ} \mathrm{C}$ for $30 \mathrm{~s}$, annealing at $66-50^{\circ} \mathrm{C}$ for $30 \mathrm{~s}\left(1^{\circ} \mathrm{C}\right.$ decrease in each cycle), and extension at $72{ }^{\circ} \mathrm{C}$ for $30 \mathrm{~s} ; 19$ cycles of $92{ }^{\circ} \mathrm{C}$ for $30 \mathrm{~s}, 50{ }^{\circ} \mathrm{C}$ for $30 \mathrm{~s}$ and $72{ }^{\circ} \mathrm{C}$ for $30 \mathrm{~s}$. A final extension was programmed at $72{ }^{\circ} \mathrm{C}$ for $5 \mathrm{mn}$. Fragments were analysed in an ABI 310 Genetic Analyser (Applied Biosystems). Allele scoring was done with Genotyper 2.5 software (Applied Biosystems). Observed and expected heterozygosities and paternity exclusionary power were estimated with the program cervus (Marshall et al. 1998). Linkage disequilibrium and Hardy-Weinberg equilibrium were tested with the probability test of genepop (http://wbiomed.curtin.edu.au/ genepop/index.html (Raymond \& Rousset 1995). Prob- ability of identity (PID) and probability of identity of full siblings $\left(\right.$ PID $_{\text {sib }}$ ) were estimated as described in Waits et al. 2001).

The mean number of alleles per locus among Spanish imperial eagles was 4.0 (Table 1). Expected heterozygosities ranged from 0.29 to 0.76 . Only 10 of the loci conformed to Hardy-Weinberg expectations when tested with the probability test of genepop (Table 1). Evidence for linkage disequilibrium between some pairs of loci was found in Spanish imperial eagle, but not in the eastern imperial eagle, A. heliaca (the sister species). The total exclusionary power in parentage analysis of these loci is 0.951 when both parents are unknown and 0.998 when one of them is known 
Table 2 Cross amplification of the 18 microsatellite markers in five raptor species. Some of the loci are fixed for some species. Number of individuals typed is shown in parenthesis when different from $n .{ }^{* * *}$ means no amplification product was obtained. None of the products have been sequenced

\begin{tabular}{|c|c|c|c|c|c|c|c|c|c|c|c|c|c|c|c|c|c|c|c|c|}
\hline \multirow[b]{2}{*}{ Locus } & \multicolumn{4}{|c|}{ Aquila heliaca $(n=18)$} & \multicolumn{4}{|c|}{ Aquila chrysaetos $(n=6)$} & \multicolumn{4}{|c|}{ Aquila nipalensis $(n=5)$} & \multicolumn{4}{|c|}{ Hieraaetus pennatus $(n=8)$} & \multicolumn{4}{|c|}{ Haliaeetus vociferoides $(n=8)$} \\
\hline & $\begin{array}{l}\text { Size } \\
\text { range }\end{array}$ & $\begin{array}{l}\text { No. of } \\
\text { alleles }\end{array}$ & $H_{\mathrm{O}}$ & $H_{\mathrm{E}}$ & $\begin{array}{l}\text { Size } \\
\text { range }\end{array}$ & $\begin{array}{l}\text { No. of } \\
\text { alleles }\end{array}$ & $H_{\mathrm{O}}$ & $H_{\mathrm{E}}$ & $\begin{array}{l}\text { Size } \\
\text { range }\end{array}$ & $\begin{array}{l}\text { No. of } \\
\text { alleles }\end{array}$ & $H_{\mathrm{O}}$ & $H_{\mathrm{E}}$ & $\begin{array}{l}\text { Size } \\
\text { range }\end{array}$ & $\begin{array}{l}\text { No. of } \\
\text { alleles }\end{array}$ & $H_{\mathrm{O}}$ & $H_{\mathrm{E}}$ & $\begin{array}{l}\text { Size } \\
\text { range }\end{array}$ & $\begin{array}{l}\text { No. of } \\
\text { alleles }\end{array}$ & $H_{\mathrm{O}}$ & $H_{\mathrm{E}}$ \\
\hline $\mathrm{Aa} 02$ & 139-157 & 9 & 0.72 & 0.80 & $135-141$ & 4 & 0.67 & 0.71 & $* * *$ & *** & & & $133-147$ & 4 & 0.75 & 0.68 & 131 & 1 & - & - \\
\hline $\mathrm{Aa} 04$ & $124-132$ & 4 & 0.78 & 0.65 & $122-150$ & 6 & 0.50 & 0.68 & $124-136$ & 4 & 0.20 & 0.8 & $125-131$ & $2(5)$ & 0.20 & 0.20 & 114 & $1(6)$ & - & - \\
\hline Aa11 & $244-252$ & 3 & 0.17 & 0.16 & $257-263$ & 2 & 0.33 & 0.30 & 252 & $1(4)$ & - & - & $253-261$ & $3(7)$ & 0.71 & 0.54 & $243-245$ & $2(7)$ & 0.57 & 0.44 \\
\hline Aa12 & $133-141$ & 3 & 0.44 & 0.52 & $* * *$ & $* * *$ & & & $138-150$ & 3 & 0.80 & 0.53 & 124 & 1 & - & - & $131-133$ & 2 & - & 0.23 \\
\hline Aa15 & $197-203$ & 4 & 0.56 & 0.72 & 199-201 & 2 & 0.50 & 0.41 & 196-206 & $4(3)$ & 1.00 & 0.87 & $198-204$ & 4 & 0.63 & 0.70 & 193 & $1(7)$ & - & - \\
\hline Aa26 & $147-149$ & 2 & 0.61 & 0.51 & 137-151 & 4 & 0.50 & 0.65 & $148-156$ & 4 & 0.80 & 0.78 & 148 & 1 & - & - & 133-135 & 2 & - & 0.23 \\
\hline $\mathrm{Aa} 27$ & 86-98 & 4 & 0.33 & 0.53 & $84-96$ & $3(5)$ & 0.40 & 0.38 & $88-94$ & 2 & 0.40 & 0.36 & 82 & 1 & - & - & $86-88$ & 2 & - & 0.23 \\
\hline Aa35 & $248-276$ & 11 & 0.89 & 0.88 & $230-256$ & 4 & 0.50 & 0.74 & $269-275$ & 4 & 0.80 & 0.80 & $* * *$ & $* * *$ & & & 239 & 1 & - & - \\
\hline Aa36 & $109-123$ & 5 & 0.67 & 0.76 & $92-102$ & 3 & 0.67 & 0.53 & $107-127$ & 6 & 0.80 & 0.89 & $* * *$ & $* * *$ & & & $112-114$ & 2 & 0.25 & 0.23 \\
\hline Aa39 & $184-202$ & 9 & 0.78 & 0.87 & $185-201$ & 6 & 0.67 & 0.88 & 168-192 & 5 & 0.40 & 0.53 & $179-203$ & 7 (7) & 1.00 & 0.85 & $* * *$ & $* * *$ & & \\
\hline Aa41 & $147-155$ & 3 & 0.39 & 0.53 & $151-153$ & 2 & 0.33 & 0.30 & $138-150$ & 3 & 0.80 & 0.60 & $* * *$ & $* * *$ & & & $* * *$ & $* * *$ & & \\
\hline Aa43 & $106-116$ & 5 & 0.61 & 0.70 & $106-130$ & 5 & 0.83 & 0.79 & $106-114$ & 5 & 0.80 & 0.76 & $105-111$ & 3 & 0.75 & 0.68 & $90-92$ & 2 & - & 0.23 \\
\hline Aa49 & $146-156$ & 5 & 0.61 & 0.72 & 137-149 & 4 & 0.17 & 0.80 & $130-150$ & 5 & 0.80 & 0.84 & $152-166$ & 5 & 0.38 & 0.68 & $142-146$ & 3 & 0.13 & 0.34 \\
\hline Aa50 & $215-217$ & 2 & 0.11 & 0.11 & $209-215$ & 2 & 0.33 & 0.30 & 215 & 1 & - & - & 208 & 1 & - & - & 218 & 1 & - & - \\
\hline Aa51 & $230-232$ & 2 & 0.33 & 0.41 & $232-234$ & 2 & 0.17 & 0.1 & $228-232$ & 3 & 0.60 & 0.51 & $226-236$ & 5 & 0.63 & 0.68 & 230 & $1(7)$ & - & - \\
\hline Aa53 & $125-133$ & 5 & 0.78 & 0.77 & $120-122$ & 2 & 0.17 & 0.17 & $127-137$ & 6 & 1.00 & 0.89 & $125-135$ & 4 & 0.75 & 0.66 & 118 & 1 & - & - \\
\hline Aa56 & $249-267$ & 8 & 0.89 & 0.80 & 251 & 1 & - & - & $237-255$ & 4 & 0.60 & 0.73 & 243 & 1 & - & - & 236 & 1 & - & - \\
\hline Aa57 & $107-117$ & 5 & 0.72 & 0.79 & $* * *$ & $* * *$ & & & $111-115$ & 3 & 0.60 & 0.60 & 113 & 1 & - & - & $123-125$ & 2 & 0.50 & 0.50 \\
\hline
\end{tabular}


(Marshall et al. 1998). PID and PID $_{\text {sib }}$ are $2.9 \times 10^{-11}$ and $1.8 \times 10^{-5}$, respectively.

A cross amplification test was performed in five other raptor species spanning from the sister taxon, the eastern imperial eagle (A. heliaca), two other species of the same genus, the golden eagle ( $A$. chrysaetos) and the steppe eagle (A. nipalensis) and two species from different genera, the booted eagle (Hieraaetus pennatus) and the madagascar fish eagle (Haliaeetus vociferoides) (Table 2).

The microsatellite markers reported here provide powerful tools for population genetic studies, paternity assessment and the unambigous identification of individuals for ecological and forensic applications for the Spanish imperial eagle and related species.

\section{Aknowledgements}

We are thankful to all the people and institutions that provided samples for this study. Governments of the five regions with nestling A. adalberti individuals, Miguel Ferrer, José A. Donázar, Todd Katdzner, Joe Busch and Melanie Culver provided samples. Juan Miguel Arroyo kindly helped with the genotyping of individuals. Roger Jovani improved an early draft of this manuscript. BMC is financed by a fellowship from the Government of La Rioja, Spain. This work was supported by FUNGESMA.

\section{References}

Criado J (2000) Aguila imperial ibérica: el ave del año recesita ayuda. La Garcilla, 107, 10-12.

David VA, Menotti-Raymond M (1998) Automated DNA detection with fluorescence-based technologies. In: Molecular Genetic Analysis of Populations. (ed. A R Hoelzel) pp. 337-370. Oxford University Press, Oxford.

Ferrer M (1993) El Águila Imperial, 1st edn. Quercus, Madrid. Gautschi B, Tenzer I, Muller JP, Schmid B (2000) Isolation and characterization of microsatellite loci in the bearded vulture (Gypaetus barbatus) and cross-amplification in three old world vulture species. Molecular Ecology, 9, 2193-2195.
González LM, Hiraldo F, Delibes M, Calderón J (1989) Reduction in the range of the Spanish Imperial Eagle (Aquila adalberti Brehm, 1861) since AD 1850. Journal of Biogeography, 16, 305315.

Marshall TC, Slate J, Kruuk L, Pemberton JM (1998) Statistical confidence for likelihood-based paternity inference in natural populations. Molecular Ecology, 7, 639-655.

Nesje M, Roed KH (2000a) Microsatellite DNA markers from the gyrfalcon (Falco rusticolus) and their use in other raptor species. Molecular Ecology, 9, 1438-1440.

Nesje M, Roed KH, Bell DA, Lindberg P, Lifjeld JT (2000c) Microsatellite analysis of population structure and genetic variability in peregrine falcons (Falco peregrinus). Molecular Ecology, 3, 267-275.

Nesje M, Roed KH, Lifjeld JT, Lindberg P, Steens OF (2000b) Genetic relationships in the peregrine falcon (Falco peregrinus) analysed by microsatellite DNA markers. Molecular Ecology, 9, 53-60.

Pearce JM, Fields RL, Scribner KT (1997) Nest materials as a source of genetic data for avian ecological studies. Journal of Field Ornithology, 68, 471-481.

Raymond M, Rousset F (1995) genepop (Version 1.2): population genetics software for exact tests and ecumenicism. Journal of Heredity, 86, 248-249.

Refseth UH, Fangan BM, Jakobsen KS (1997) Hybridization capture of microsatellites directly from genomic DNA. Electrophoresis, 18, 1519-1523.

Sambrook E, Fritsch F, Maniatis TC (1989) Molecular Cloning. Spring Harbor Press, Cold Spring Harbor, New York.

Sarno RJ, David VA, Franklin WL, O’Brien SJ, Johnson WE (2000) Development of microsatellite markers in the guanaco, Lama guanicoe: utility for South American camelids. Molecular Ecology, 9, 1919-1952.

Strausberger BM, Ashley MV (2001) Eggs yield nuclear DNA from egg-laying female cowbirds, their embryos and offspring. Conservation Genetics, 2, 385-390.

Taberlet P, Waits LP, Luikart G (1999) Noninvasive genetic sampling: look before you leap. Trends in Ecology and Evolution, 14, 323-327.

Waits LP, Luikart G, Taberlet P (2001) Estimating the probability of identity among genotypes in natural populations: cautions and guidelines. Molecular Ecology, 10, 249-256. 